\title{
SIDR Experiment: new results and new equipment
}

\section{George Bashindzhagyan}

M.V. Lomonosov Moscow State University (RU)

\section{Virgil Barnes}

Purdue University (IN, USA)

\section{Ephraim Fischbach*}

Purdue University (IN, USA)

E-mail: ephraimepurdue.edu

\section{Gali Garipov}

M.V. Lomonosov Moscow State University (RU)

\section{Gagik Hovsepyan}

Yerevan Physics Institute (AM)

\section{Natalia Korotkova}

M.V. Lomonosov Moscow State University (RU)

\section{Levon Poghosyan}

Yerevan Physics Institute (AM)

\section{Nikolai Sinev}

University of Oregon (OR, USA)

The general idea of the SIDR project is to continuously monitor the decay rates of several identical ${ }^{90} \mathrm{Sr}$ radioactive sources [1,2]. Additionally, several short-term experiments were performed to look for possible correlations between decay rate changes and environmental conditions. Interesting results have been obtained in the experiment by moving filters above and below the decay modules. A new rate measuring setup utilizing internal power supplies and temperature stabilization has been incorporated for the field experiments.

36th International Cosmic Ray Conference -ICRC2019-

July 24th - August 1st, 2019

Madison, WI, U.S.A.

${ }^{*}$ Speaker. 


\section{Introduction}

The main goal of the SIDR experiment is to measure the decay rate of several identical ${ }^{90} \mathrm{Sr}$ sources over a long time, and to check for a possible correlation between decay rates and the distance between the Earth and the Sun. Additionally, the high decay rates of the sources that were employed (up to 200,000 decays per second (dps) ) allows us to detect short-term decay rate changes, and their correlations with different natural and technogenic influences. To detect small decay rate variations new very stable electronics, based on silicon detectors, have been created. However, even if small decay rate changes are detected, we cannot be sure whether these changes are the result of an external influence, or of electronics instability. Therefore, several interesting short-term experiments have been performed. A special automatic system has been created which repeatedly changed the surrounding space. This has been used to search for possible decay rate violations which have been precisely measured.

\section{New experimental setup and interesting results}

The high decay rates of our sources (up to 200,000 dps) allows small changes in decay rates to be detected. However, the readout electronics are sensitive to small temperature and supply voltage changes. This results in a shift of the detection threshold of individual channels, thereby changing the number of recorded decays per second, simulating variation in the decay rate.

The conventional way to deal with these "instrumental" effects is to stabilize the temperature and voltage of the power sources. For this purpose, new experimental equipment, with autonomous thermally insulated modules powered by a battery source, have been developed. Scintillators, previously used for decay electron detection, have been replaced with modern stable silicon detectors. However, the problem with detection of slight variations in decay rates still remains. Hence, it is not possible to guarantee that the recorded small changes in decay rates are caused by external factors, and not by the drift of the electronics threshold. To overcome this problem, an experiment with a moving filter has been carried out. A specially designed automatic device artificially changes the environment, alternately placing filters made of different materials and different thickness (1-5 $\mathrm{cm}$ ) above or below the ${ }^{90} \mathrm{Sr}$ radioactive sources.

The decay rate is measured over a strictly fixed time interval of $100 \mathrm{~s}$ with or without a filter. Such a short measurement interval is chosen in order to minimize the impact of any slow violations in the counting rate, regardless of whether the source of these changes is an instrumental effect (temperature, voltage, etc.) or the influence of slowly changing external conditions.

A special light trolley, which can carry any filter (Fig. 1), moves the filter from the left or right position to the opposite one. The movement itself takes approximately 10 seconds, after which the decay rates of the left and right sources are measured for exactly $100 \mathrm{~s}$. The full measurement cycle extends for $240 \mathrm{~s}$, during which time the filter appears either under the left source (channel 1) or above the right source (channel 2). It should be noted that channel 1 had better time stability than channel 2. However, even for the worst measurement in channel 2, the general drift of the measurement did not exceed $1 \%$.

The results of one of the experiments, where a $5 \mathrm{~cm}$ thick stack of $15 \times 20 \mathrm{~cm}$ paper was used as a filter, are shown in Fig.2. Open points refer to measurements without a filter, and solid points 

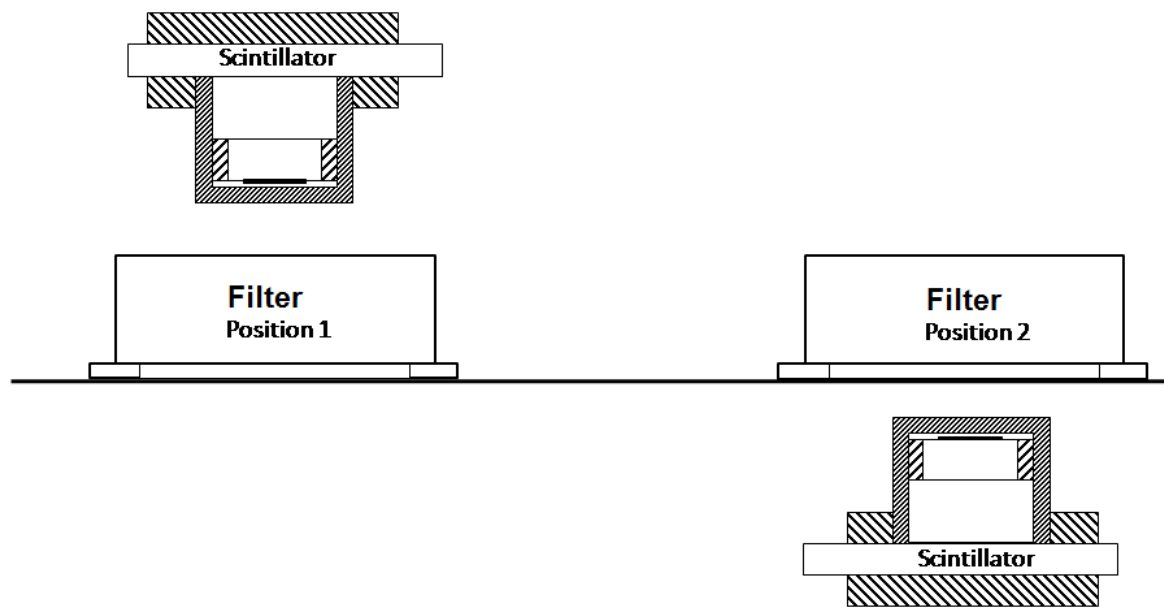

Figure 1: Experiment with moving filter.

refer to the measurements with a filter, which was placed alternately under channel 1 or above channel 2. It is clearly seen that the presence of a filter under source number 1 reduces the counting rate by 70 counts/s in a background whose total rate is 100000 counts/s, or about $0.07 \%$.

Many other filters including lead and water have been tested, however, most of them affect the decay rate much less than the paper filters. To check a possible influence of cosmic rays, the number of registered events has been measured without a source but with all the other equipment. Cosmic ray influence was less than 8 counts per second and did not depend on appearance of the filter.

\section{Conclusion}

This experiment is potentially important because it suggests that the ${ }^{90} \mathrm{Sr}$ decay rate can be changed (decreased) with a simple filter positioned below the source. This implies that radioactive decay, at least for ${ }^{90} \mathrm{Sr}$, may not be a completely internal process. It would be very interesting to learn carefully what kind of influence can speed up or decrease the process. There is a chance that decays can be initiated by unknown particles or rays whose energies or cross sections are too small to create an interaction with a stable regular nucleus, but large enough to slightly perturb an unstable nucleus.

\section{References}

[1] G.Bashindzhagyan, V.Barnes, E.Fischbach, G.Hovsepyan, N.Korotkova, L.Poghosyan, N.Sinev, SIDR experiment status and first results, in proceedings of 35th International Cosmic Ray Conference, POS (ICRC2017) 050 (2017).

[2] G.Bashindzhagyan, V.Barnes, E.Fischbach, G.Hovsepyan, N.Korotkova, M.Merkin, N.Sinev, A.Voronin, Solar Influence on Decay Rate (SIDR) Experiment, in proceedings of 34th International Cosmic Ray Conference, PoS ( ICRC2015) 136 (2015). 

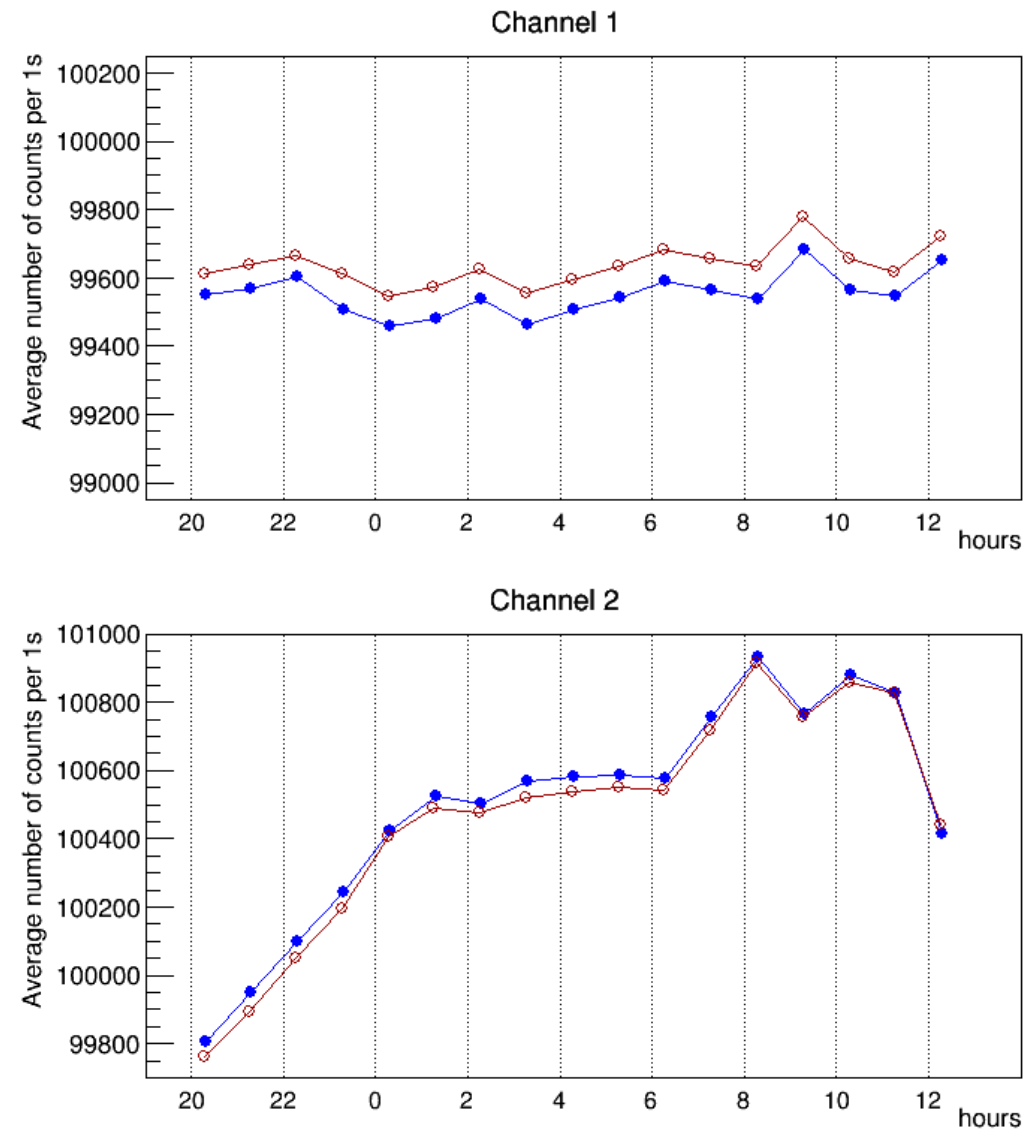

Figure 2: One of the interesting very results obtained in the experiment with moving filter. The upper plot shows the results for more stable channel 1 . It clearly demonstrates that ${ }^{90} \mathrm{Sr}$ source positioned in the steel container systematically changes its decay rate when a paper filer appeared under the container. Less stable channel 2 does not show such clear changes when the same filter appears above container 2. 